\title{
Fatty Streak Formation Occurs in Human Fetal Aortas and is Greatly Enhanced by Maternal Hypercholesterolemia

\author{
Intimal Accumulation of Low Density Lipoprotein and its Oxidation Precede Monocyte Recruitment into
} Early Atherosclerotic Lesions
}

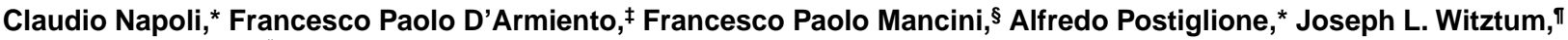 \\ Giuseppe Palumbo," and Wulf Palinskit \\ $*$ Department of Clinical and Experimental Medicine, ${ }^{\ddagger}$ Department of Human Pathology, ${ }^{\S}$ Department of Biochemistry and Medical

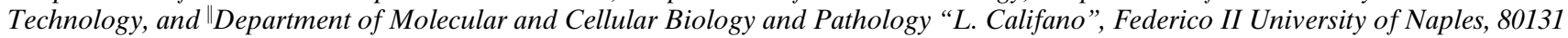 \\ Naples, Italy; and "Department of Medicine, University of California, San Diego, La Jolla, California 92093
}

\begin{abstract}
To determine whether oxidized LDL enhances atherogenesis by promoting monocyte recruitment into the vascular intima, we investigated whether LDL accumulation and oxidation precede intimal accumulation of monocytes in human fetal aortas (from spontaneous abortions and premature newborns who died within $12 \mathrm{~h}$; fetal age $6.2 \pm 1.3$ mo). For this purpose, a systematic assessment of fatty streak formation was carried out in fetal aortas from normocholesterolemic mothers $(n=22)$, hypercholesterolemic mothers $(n=33)$, and mothers who were hypercholesterolemic only during pregnancy $(n=27)$. Fetal plasma cholesterol levels showed a strong inverse correlation with fetal age $(R=-0.88, P<0.0001)$. In fetuses younger than $6 \mathrm{mo}$, fetal plasma cholesterol levels correlated with maternal ones $(R=0.86, P=0.001)$, whereas in older fetuses no such correlation existed. Fetal aortas from hypercholesterolemic mothers and mothers with temporary hypercholesterolemia contained significantly more and larger lesions $\left(758,651 \pm 87,449\right.$ and $451,255 \pm 37,448 \mu \mathrm{m}^{2}$ per section, respectively; mean \pm SD) than aortas from normocholesterolemic mothers $\left(61,862 \pm 9,555 \mu \mathrm{m}^{2} ; P<0.00005\right)$. Serial sections of the arch, thoracic, and abdominal aortas were immunostained for recognized markers of atherosclerosis: macrophages, apo $\mathrm{B}$, and two different oxidation-specific epitopes (malondialdehyde- and 4-hydroxynonenal-lysine). Of the atherogenic sites that showed positive immunostaining for at least one of these markers, $58.6 \%$ were established lesions containing both macrophage/foam cells and oxidized
\end{abstract}

Parts of this study were presented at the XIX. Congress of the European Society of Cardiology, Stockholm, Sweden, August 24-28, 1997 and the Royaumont Satellite of the XI. International Symposium on Atherosclerosis, Paris, France, October 2-4, 1997.

Address correspondence to Wulf Palinski, M.D., Department of Medicine, 0682, University of California, San Diego, 9500 Gilman Drive, MTF 110, La Jolla, CA 92093. Phone: 619-534-7818; FAX: 619534-2005; E-mail: wpalinski@uscd.edu; or Claudio Napoli, M.D., Department of Clinical and Experimental Medicine, Federico II University of Naples Via S. Pansini 5, 80131 Naples, Italy. Phone/FAX: +39-81-560-3990.

Received for publication 2 July 1997 and accepted in revised form 24 September 1997.

J. Clin. Invest.

(c) The American Society for Clinical Investigation, Inc. 0021-9738/97/12/2680/11 \$2.00

Volume 100, Number 11, December 1997, 2680-2690

http://www.jci.org
LDL (OxLDL). $17.3 \%$ of all sites contained only native LDL, and $13.3 \%$ contained only OxLDL without monocyte/ macrophages. In contrast, only $4.3 \%$ of sites contained isolated monocytes in the absence of native or oxidized LDL. In addition, $6.3 \%$ of sites contained LDL and macrophages but few oxidation-specific epitopes. These results demonstrate that LDL oxidation and formation of fatty streaks occurs already during fetal development, and that both phenomena are greatly enhanced by maternal hypercholesterolemia. The fact that in very early lesions LDL and OxLDL are frequently found in the absence of monocyte/macrophages, whereas the opposite is rare, suggests that intimal LDL accumulation and oxidation contributes to monocyte recruitment in vivo. (J. Clin. Invest. 1997. 100:2680-2690.) Key words: arteriosclerosis - lipoprotein oxidation - macrophages $\bullet$ hypercholesterolemia $\bullet$ fetal development

\section{Introduction}

The recruitment of circulating monocytes into the vascular intima and their subsequent transformation into macrophage/ foam cells are key elements of the initiation of atherosclerosis. Little is known, however, about the factors responsible for and the mechanisms involved in monocyte recruitment. Oxidative modification of LDL is thought to play an important role in atherogenesis (for review see references 1-3). Lipid peroxidation products generated during LDL oxidation are chemotactic for both monocytes and T cells, and inhibit the motility of macrophages, thus trapping them in the intima. In addition, even minimally modified LDL (i.e., LDL oxidized to an extent that does not suffice for recognition by scavenger receptors) may enhance expression of adhesion molecules and cytokines by endothelial cells (3). In turn, antioxidants inhibit upregulation of some of these genes, e.g., vascular cell adhesion molecule 1 , both in vitro and in vivo (4-6). Therefore, it has been hypothesized that increased formation of OxLDL ${ }^{1}$ in the vascular intima is responsible for monocyte recruitment. OxLDLmediated events would then induce the phenotypic transformation of monocytes into macrophages, and subsequent uptake of OxLDL by scavenger receptors would result in foam cell formation. Other atherogenic effects of OxLDL, e.g., its cytotoxicity, interference with vascular relaxation in response to nitric oxide (7), or platelet-activating factor-dependent proaggregatory effects (8), are more likely to influence later stages of atherogenesis.

1. Abbreviations used in this paper: 4-HNE, 4-hydroxynonenal; MDA, malondialdehyde; OxLDL, oxidized LDL. 
In vivo evidence for the atherogenicity of OxLDL was provided by the fact that several powerful antioxidants, e.g. probucol, butylated hydroxytoluene, and diphenyl-phenylenediamine, significantly reduced progression of atherosclerosis in rabbits (9-11), primates (12), and mice (13). On the other hand, less powerful antioxidants such as vitamin E yielded inconsistent results in animal models (14-16). Evidence for the antiatherogenic effect of antioxidants in humans is still inconclusive (17-21).

Immunocytochemistry with antibodies to different epitopes of OxLDL has confirmed the presence of OxLDL in atherosclerotic lesions of various animal models (22-26) and in human lesions $(27,28)$. However, the temporal sequence of LDL penetration, LDL oxidation, and intimal monocyte accumulation have never been systematically studied in very early stages of lesion formation. This is due in part to the fact that the aortic intima of most animal models (in which lesion formation could be induced under well-controlled conditions) consists of little more than the endothelial monolayer. Thus, before intimal accumulation of macrophage/foam cells occurs it is difficult to define sites at which lesion formation has been initiated. The lack of intimal tissue does not favor an immunocytochemical study of native and oxidized LDL in these models, at least by light microscopy. In contrast, human arteries are very suitable for this kind of analysis because of the presence of adaptive intimal thickening at lesion-prone sites (29).

To determine the temporal sequence of intimal accumulation and oxidation of LDL and intimal accumulation of monocytes, we examined human fetal aortas for the presence of LDL, oxidation-specific epitopes, and macrophage/foam cells in early lesions. Because hypercholesterolemia is a well-recognized risk factor of atherogenesis and because it enhances LDL oxidation in humans (30-32), we used fetal aortas from hypercholesterolemic mothers and mothers with temporary hypercholesterolemia during pregnancy in addition to normocholesterolemic mothers. In doing so, this study also provides the first systematic morphometric assessment of fatty streak development in fetal aortas, and yields information on the role of maternal and fetal hypercholesterolemia in fetal fatty streak formation.

\section{Methods}

Human subjects. Aortas $(n=82)$ were obtained from spontaneously aborted fetuses $(n=35)$ and premature newborns who died within $12 \mathrm{~h}$ of birth $(n=47)$. The mean fetal age was $6.2 \pm 1.3 \mathrm{mo}$. Because there was no difference in age between the spontaneously aborted fetuses and the premature newborns, all of these aortas will be termed "fetal aortas." Mothers had presented with acute signs of imminent birth/abortion or with birth in progress. Fetuses and premature newborns who died in pediatric intensive care were routinely subjected to autopsy at the Department of Human Pathology of the Federico II University of Naples. Causes of spontaneous abortion and premature death included trauma, eclampsia, and fetal genetic defects (often associated with cerebral malformations). Fetuses from mothers with diseases affecting hematopoiesis or the immune system (including HIV) were excluded from the study. To reduce potential oxidative artifacts, only aortas that were obtained within $3.5 \mathrm{~h}$ after death were used. Fetuses were classified based on maternal plasma cholesterol levels that were determined at admission to the hospital. In addition, a detailed history was obtained to ascertain past plasma cholesterol levels before and during pregnancy. Maternal and fetal plasma cholesterol levels in venous blood were determined in a routine clinical laboratory by an automated enzymatic procedure using kits from Boehringer Mannheim (Mannheim, Germany). To investigate the correlation of fetal cholesterol levels with maternal cholesterol levels and fetal age, only data from fetal blood samples obtained before death were used. Lipoperoxide levels in EDTA-containing plasma were evaluated spectrophotometrically using the LPO kit (Kamiya Biomedical Company, Thousand Oaks, CA) as previously described $(31,32)$.

Of the mothers, 22 had normal plasma cholesterol levels (total plasma cholesterol $\leq 185-200 \mathrm{mg} / \mathrm{dl}$, depending on age; normocholesterolemia), 27 were hypercholesterolemic only during pregnancy (temporary hypercholesterolemia), and 33 were hypercholesterolemic both before and during pregnancy (hypercholesterolemia; see Table I). The three groups showed no significant differences in maternal race, age $(27 \pm 5,28 \pm 6$, and $28 \pm 5 \mathrm{yr}$ in the groups with normocholesterolemia, temporary hypercholesterolemia, and hypercholesterolemia, respectively), and smoking habits $(33,21$, and $28 \%$ of subjects were current smokers in the three groups). The mean age of the fetuses of all three groups was also similar, and within each group the fetal body mass index was proportional to the stage of pregnancy (data not shown).

The protocol of the study was approved by the Human Ethical Committee of Federico II University, Naples.

Preparation of aortic sections. The fetal aorta was exposed, the branching arteries were cut off, and loose adventitial tissue was removed in situ. The vessel was then cut open, thoroughly washed with cold sterile PBS containing 2 mM EDTA to remove adherent blood cells, and placed in ice-cold PBS containing $50 \mu \mathrm{M}$ butylated hydroxytoluene, $0.001 \%$ aprotinin, $50 \mu \mathrm{M}$ EDTA, and $0.008 \%$ chloramphenicol equilibrated with nitrogen to reduce spontaneous lipid peroxidation in the intima. The aorta was divided into arch, thoracic, and abdominal segments. For the first 29 aortas, all segments were immersed in OTC medium, flash-frozen in liquid nitrogen, and sectioned with a cryotome. Approximately 60 frozen sections were cut from each segment and stained with oil red O. Of these, 30 step sections each were used for morphometric evaluation of lipid-containing lesions in the arch, thoracic, and abdominal segments (see below). Thus, quantitation of lesion areas and distribution was based on 90 sections per aorta. Additional sections were stained with Alcian blue to determine the density of smooth muscle cells in the media, a measure of tissue maturity. For the first 29 aortas, only morphometric analysis was performed. For the remaining 53 aortas, the arch, thoracic, and abdominal segments were further subdivided into two parts. One part was frozen and analyzed as described above. The second part was fixed in buffered $10 \%$ formalin, paraffin-embedded, and 12-15 serial sections $(5-7 \mu \mathrm{m}$ thick) were prepared for immunocytochemistry.

Measurement of lesion sizes and maturity. To determine the size of lesions in fetal aortas, 90 sections each from all 82 aortas were stained with oil red $\mathrm{O}$ and counterstained with hematoxylin (33). Lesions were then photographed, and the photographic slides in turn were scanned to obtain electronic images using a Macintosh Color One Scanner (Apple Computer, Cupertino, CA). All sections, blinded for the classification of the fetal aorta, were analyzed by F.P. D'Armiento using an Orthoplan microscope equipped for microphotography (Leitz, Wetzlar, Germany). The following parameters were determined by computer-assisted image analysis, using Ofoto software T1-2 version 2.0 (Apple Computer): (a) the greatest intimal/medial ratio of normal adaptive intimal thickenings and atherosclerotic lesions (this was to provide a measure of the degree of intimal thickening occurring in fetal aortas); (b) the number of oil red $\mathrm{O}$-positive lipid accumulations in each section (because the same adaptive intimal thickening frequently showed more than one area staining with oil red $\mathrm{O}$, no attempt was made to define and count individual lesions; instead, the entire aortic section was counted as lesioned when at least one area of lipid accumulation was present); (c) the cumulative surface area of all oil red O-positive areas per section, determined in all 90 sections of each aorta-30 each from the arch, thoracic, and abdominal segments (measuring the areas of oil red $\mathrm{O}$-positive 
lipid accumulation is preferred to measuring the entire intimal area because it allows one to assess the onset of lesion formation in normal adaptive intimal thickenings, and because it avoids the error associated with determining the medial delimitation of very small lesions); and $(d)$ the surface area of the single largest lesion encountered in each section. This was to provide a measure of lesion formation using a more traditional definition of lesions (i.e., areas with increased intimal/ medial ratio containing both lipid accumulations and macrophage/ foam cells). Intercostal, mesenteric, and renal artery branch points were included in the evaluation of the thoracic and abdominal segments of the aorta, but were not assessed separately.

To obtain a measure of the maturity of the fetal aortas, the density of smooth muscle cells in the media was measured in 8 sections each from 58 fetal aortas $(6.0 \pm 1.4 \mathrm{mo})$. These sections were stained with $\mathrm{Al}-$ cian blue using a standard procedure (34), and the number of stained cell nuclei per area unit of the media was determined with the image analysis system described above. For comparison, nuclear density was also measured in equivalent areas of the aorta of 6 adults $(28 \pm 4 \mathrm{yr})$.

Immunocytochemistry. Duplicate serial sections of the fixed and paraffin-embedded segments of the arch, thoracic, and abdominal sections of each fetal aorta were immunostained with the following antibodies: (a) MDA2 and NA59 at a dilution of 1:500 $(1 \mu \mathrm{g} / 150 \mu \mathrm{l})$, two murine monoclonal antibodies specific for malondialdehyde (MDA)-lysine and 4-hydroxynonenal (4-HNE)-lysine epitopes, respectively. These antibodies were generated by immunization of mice with homologous MDA-LDL and 4-HNE-LDL, and recognize the above epitopes on OxLDL as well as on other adducts between proteins and lipid peroxidation products. In other words, these antibodies recognize oxidation-specific epitopes, but are not specific for oxidized LDL (35); (b) NP1533975, a mouse monoclonal antibody $\left(\mathrm{IgG}_{1}\right)$ to human apo B (1:500 dilution; Boehringer Mannheim Italia, Monza, Italy). This antibody recognizes apo B, but staining with this antibody does not exclusively represent native, nonoxidized LDL because the apo B epitope is only destroyed by very extensive oxidation; and (c) HAM-56, a monoclonal antibody generated against human macrophages that also recognizes monocytes, but not $\mathrm{B}$ and $\mathrm{T}$ lymphocytes (1:500 dilution; Axcel Accurate, Westbury, NY) (36). To ensure that all monocytes are recognized, a number of serial sections of lesions/lesion-prone areas containing no or only isolated monocytes recognized by HAM-56 were also stained with a second monoclonal antibody to monocyte/macrophages (1:500 dilution; antiCD11b, Boehringer Mannheim). This antibody is specific for the complement receptor, a membrane glycoprotein that occurs on monocytes, but not on $\mathrm{B}$ and $\mathrm{T}$ cells, and that is preserved during monocyte differentiation.

Immunocytochemistry was performed using an avidin-biotin peroxidase method. Before immunostaining, endogenous peroxidase activity potentially present in the aortic tissue was quenched by a 10-min incubation of the sections at $4^{\circ} \mathrm{C}$ with $0.3 \%$ hydrogen peroxide in PBS containing $0.5 \mu \mathrm{M}$ butylated hydroxytoluene. Slides were washed twice with PBS, and were incubated for $30 \mathrm{~min}$ with normal horse serum (Dako Corp., Carpinteria, CA) and for $60 \mathrm{~min}$ at room temperature with the primary antibody. Slides were then thoroughly rinsed, incubated for $30 \mathrm{~min}$ with biotinylated horse anti-mouse IgG (1:500 dilution; Dako Corp.), washed, and incubated for $30 \mathrm{~min}$ with avidinbiotin peroxidase (Dako Corp.). Antibodies bound were visualized by 20-min incubation with 3,3-diaminobenzidine tetrahydrochloride. Because of the large number of sections stained and the need for identical staining conditions, all immunocytochemistry was performed using an automated tissue staining machine (TM500; BioTek Solutions, Baltimore, MD) that provided consistent temperature, reagent volume, humidity, and incubation times.

Serial sections immunostained with our panel of antibodies were used for two different assessments. We first determined the percentage of sections showing staining for monocyte/macrophages, apo B, and MDA-lysine or 4-HNE epitopes, irrespective of whether the staining was located in the same area of the section. We then investigated the temporal sequence of events in early atherogenesis follow- ing a different protocol. For this purpose, an atherogenic site was defined as a specific area of thickened intima immunostaining with at least one of the antibodies. Spatially separated areas in the same adaptive thickening were treated as distinct sites. Corresponding areas of the serial sections were checked for staining with all other antibodies, and atherogenic sites were classified depending upon whether they contained $(a)$ only monocyte/macrophages; $(b)$ only native LDL; (c) LDL and isolated monocyte/macrophages; (d) OxLDL (one or both oxidation-specific epitopes and apo B but no macrophages); or (e) both OxLDL and monocytes, macrophages, or foam cells.

Statistical analysis. Results were analyzed by one-way ANOVA followed by Bonferroni's corrected $t$ test. A $P<0.05$ was considered significant. Numerical data obtained from immunocytochemistry were analyzed for mean, variance, standard deviation, kurtosis and skew. Correlations were determined by linear regression analysis.

\section{Results}

Maternal and fetal plasma cholesterol levels. The maternal plasma cholesterol and triglyceride levels are shown in Table I. In all three groups an increase in plasma cholesterol levels was observed during pregnancy, but this increase was significant only in the two hypercholesterolemic groups. During pregnancy, plasma cholesterol levels of the two hypercholesterolemic groups were similar $(325 \pm 44$ vs. $385 \pm 50 \mathrm{mg} / \mathrm{dl}$; NS), whereas the difference between these groups and the normocholesterolemic group $(175 \pm 20 \mathrm{mg} / \mathrm{dl})$ was highly significant as expected. Fetal plasma cholesterol values before death were determined in a subgroup of 44 cases $(54 \% ; 17$ from the normocholesterolemic, 16 from the temporary hypercholesterolemic, and 11 from the hypercholesterolemic group), ages $6.4 \pm 0.6 \mathrm{mo}$, averaging $185 \pm 54 \mathrm{mg} / \mathrm{dl}$. Individual values are shown in Fig. $1 A$. When data from the entire subgroup were analyzed together, fetal cholesterol levels showed only a weak correlation with maternal ones $(R=0.37, P<0.02)$. This result is in agreement with the well-known fact that maternal and fetal lipoprotein levels are generally not correlated at birth (38). Average plasma cholesterol levels of fetuses from normocholesterolemic and hypercholesterolemic mothers were similar $(196 \pm 32$ and $179 \pm 45 \mathrm{mg} / \mathrm{dl}$, respectively). Plasma cholesterol levels of the younger fetuses ( $<6$ mo of age, $n=10$ ), however, were significantly higher than those of older fetuses

Table I. Maternal Plasma Cholesterol and Triglyceride Levels

\begin{tabular}{cccc}
\hline & $\begin{array}{c}\text { Normocho- } \\
\text { lesterolemia }\end{array}$ & $\begin{array}{c}\text { Hypercholester- } \\
\text { olemia during } \\
\text { pregnancy }\end{array}$ & $\begin{array}{c}\text { Hypercho- } \\
\text { lesterolemia }\end{array}$ \\
\hline & $n=22$ & $n=27$ & $n=33$ \\
$\begin{array}{c}\text { Plasma cholesterol } \\
\text { (mg/dl) }\end{array}$ & & & \\
$\quad \begin{array}{l}\text { before pregnancy } \\
\text { during pregnancy }\end{array}$ & $155 \pm 28$ & $178 \pm 30$ & $292 \pm 41^{*}$ \\
$\begin{array}{c}\text { Plasma triglycerides } \\
\text { (mg/dl) }\end{array}$ & $175 \pm 20$ & $325 \pm 44^{\ddagger \S}$ & $385 \pm 50^{\ddagger \S}$ \\
$\quad \begin{array}{l}\text { before pregnancy } \\
\text { during pregnancy }\end{array}$ & $165 \pm 20$ & $155 \pm 21$ & $161 \pm 28$ \\
\hline
\end{tabular}

${ }^{*} P<0.05$ vs. normocholesterolemic mothers and mothers with temporary hypercholesterolemia during pregnancy; ${ }^{\ddagger} P<0.05$ vs. respective values before pregnancy; ${ }^{\S} P<0.05$ vs. normocholesterolemic mothers. 

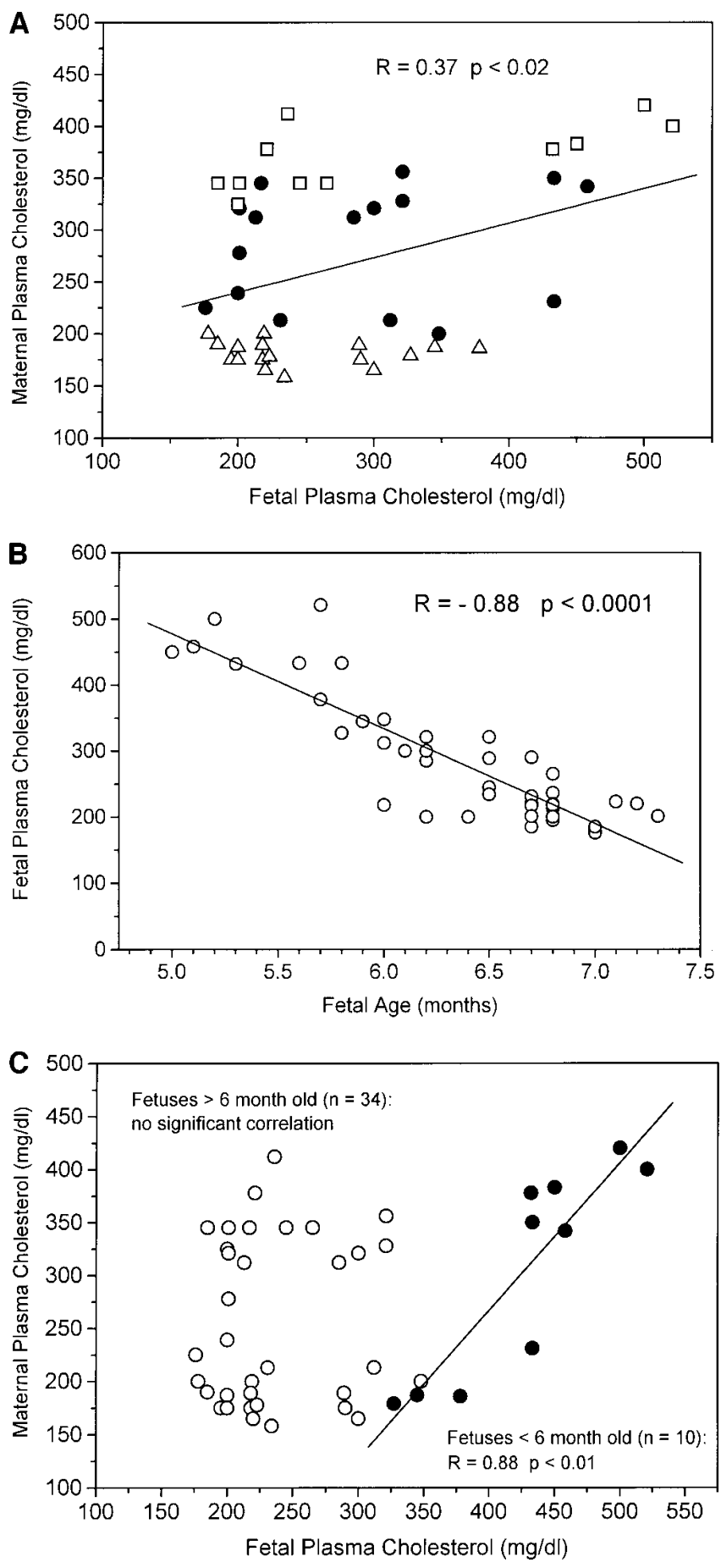

Figure 1. (A) Correlation between fetal and maternal plasma cholesterol. Maternal classification (normocholesterolemia, temporary hypercholesterolemia or hypercholesterolemia) is indicated by the symbols, but regression was calculated from pooled data. Data represent a subset of 44 cases (54\%), mean age $6.37 \pm 0.6 \mathrm{mo}$, for which fetal cholesterol levels before death were available. Open triangles, normocholesterolemia; closed circles, temporary hypercholesterolemia; open squares, hypercholesterolemia. (B) Correlation between fetal age and fetal plasma cholesterol level in the same group of 44 cases. $(C)$ Correlation between fetal and maternal plasma cholesterol. Data are the same as in $A$, but correlation was analyzed separately for younger $(<6$ mo old $)$ and older fetuses.
( $427 \pm 62$ vs. $237 \pm 48 \mathrm{mg} / \mathrm{dl}, P<0.0001)$. When fetal cholesterol levels were plotted against fetal age (Fig. $1 B$ ), a highly significant inverse correlation was established $(R=-0.88, P<$ $0.0001)$. When we reexamined the correlation between fetal and maternal cholesterol levels by fetal age (i.e., irrespective of the maternal classification), we found an excellent correlation between fetal and maternal cholesterol levels in fetuses younger than 6 mo $(R=0.88, P<0.01)$, whereas no correlation existed for fetuses 6 mo old or older (Fig. $1 C$ ). Plasma lipoperoxide levels were $0.63 \pm 0.24 \mu \mathrm{M}$ in normocholesterolemic mothers, $0.71 \pm 0.22 \mu \mathrm{M}$ in mothers with temporary hypercholesterolemia, and $0.85 \pm 0.27 \mu \mathrm{M}$ in hypercholesterolemic mothers $(P<0.05$ vs. normocholesterolemic mothers $)$. As expected, there was a positive correlation between cholesterol levels and lipoperoxide levels in the two hypercholesterolemic groups $(R=0.65, P<0.01 ; R=0.77, P<0.003$ in mothers with temporary or continuous hypercholesterolemia, respectively), as well as in pooled data from all three groups $(R=0.58, P<0.05)$.

Maturity of fetal aortas. Morphometric analysis of the media of 58 fetal aortas from all three groups showed 755 123 smooth muscle cell nuclei/ $\mathrm{mm}^{2}$. Smooth muscle cell density was significantly smaller than that determined in the aortic media of adults $(1,005 \pm 110)$ and from comparable values reported in literature $(37)$. Data were not normally distributed $($ skew $=2.12)$ and were therefore analyzed by Wilcoxon test. When the fetal arch, thoracic, and abdominal aorta were compared, no significant differences in nuclear density were found $(P=0.65)$.

Extent of fatty streak formation in fetal aortas. Morphometric analysis of fetal aortas was performed in 30 oil red O-stained sections each from the arch, thoracic, and abdominal segments of all 82 fetal aortas. At this very early stage, the amount of lipid accumulated in the intima often does not substantially increase the intimal area, which is often determined primarily by an underlying adaptive intimal thickening (in the absence of lipid accumulation and foam cell formation, adaptive intimal thickenings are generally not considered lesions, even though they are found at predilection sites of atherosclerosis). Therefore, lesion formation was assessed in terms of the number and area of oil red $\mathrm{O}$-positive intimal lipid accumulations (Fig. 2) rather than in terms of the entire intimal area. To our surprise, large numbers of lipid accumulations were found in all aortas, including fetal aortas from normocholesterolemic mothers. When results were expressed as percent of sections containing at least one such area (Fig. $3 A$ ), 63.3\% of aortas were affected in the normocholesterolemic group vs. 77.8 and $75.6 \%$ in the hypercholesterolemic group and the group with temporary hypercholesterolemia during pregnancy, respectively. The differences between the two hypercholesterolemic groups and the normocholesterolemic group were significant in the arch and abdominal segments $(P<0.05)$, but not in the thoracic aorta. Contrary to expectation, the percentages of positive sections of the thoracic segments were not much smaller than those of the rest of the aorta. The numbers of lipid-rich areas per section (actual numbers shown in the bars in Fig. $3 A$ ) were much greater in both hypercholesterolemic groups than in controls, and this difference was most pronounced in the abdominal aorta where atherosclerosis is most extensive in adults and adolescents (39).

We then compared the area of the intimal lipid accumulations, measuring both the single largest lesion in each section (Fig. $3 \mathrm{~B}$ ) and the cumulative area of all oil red O-positive ar- 

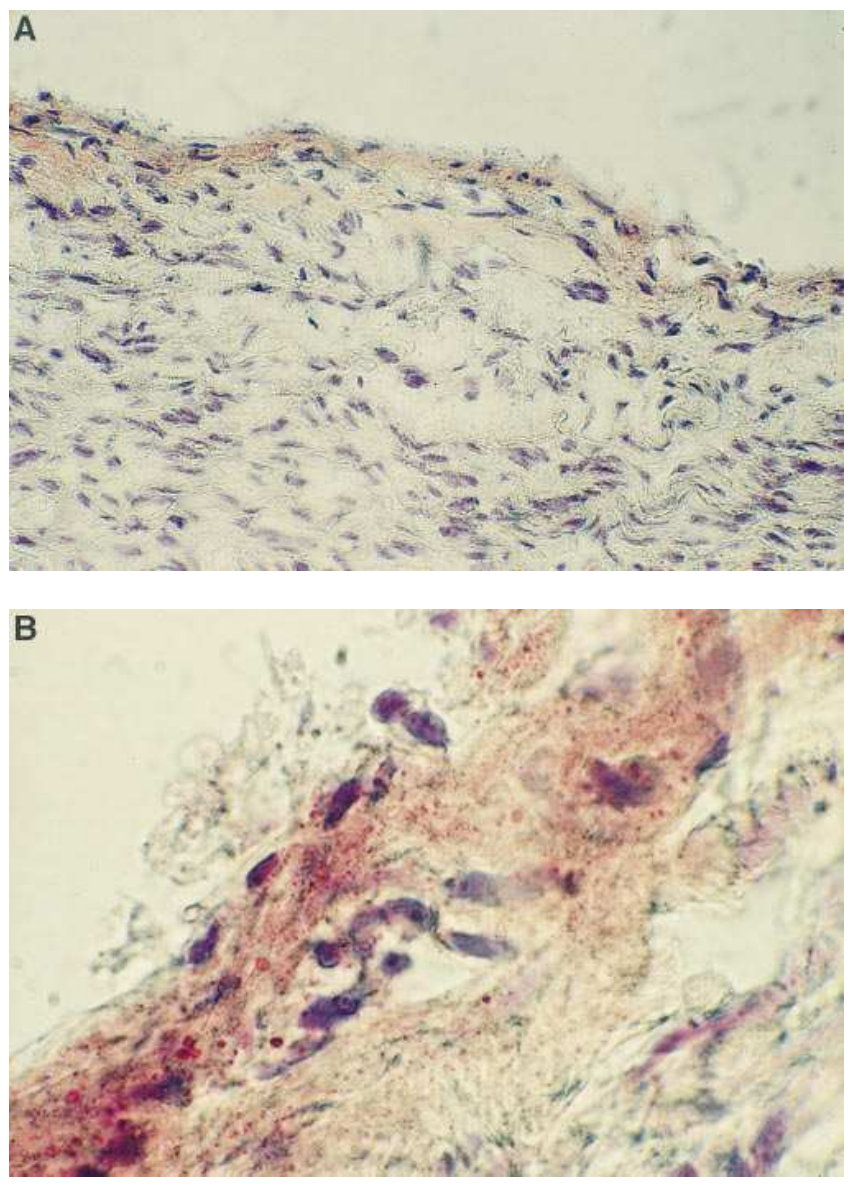

Figure 2. Presence of intimal lipid accumulations in fetal aortas. Frozen sections were prepared from the arch, thoracic, and abdominal segments of all 82 fetal aortas and stained with oil red $\mathrm{O}$, as described in Methods. These sections were used to determine various measures of lesion formation, including the area of lipid accumulations and the intimal/medial ratio of lesions (see Fig. 3). Representative examples are shown here. $(A)$ An adaptive intimal thickening with signs of beginning lipid accumulation. $(B)$ Higher magnification of a fatty streak containing substantial amounts of lipid (magnification: $A, 562.5 \times ; B$, $2250 \times)$.

eas (Fig. $3 C$ ). As shown in Fig. $3 B$, fetal aortas from both groups of hypercholesterolemic mothers contained dramatically greater lesion areas than did aortas from the normocholesterolemic group. Differences were significant at $P<0.005-$ 0.001 in the abdominal aorta and the aortic arch (as well as in the entire aorta), whereas the absolute lesion size in the thoracic aorta was small, and differences between groups were not significant. Comparison of the cumulative areas of all intimal lipid accumulations (Fig. $3 C$ ) yielded essentially the same result. Note that the values of the hypercholesterolemic group were consistently higher than those of the group with temporary hypercholesterolemia during pregnancy, even though, ex-

Figure 3. Quantitation of lesions in 82 fetal aortas from normocholesterolemic mothers, mothers with temporary hypercholesterolemia during pregnancy, and hypercholesterolemic mothers. A total of 90 sections of each aorta (30 each from the arch, thoracic, and abdomi-
A

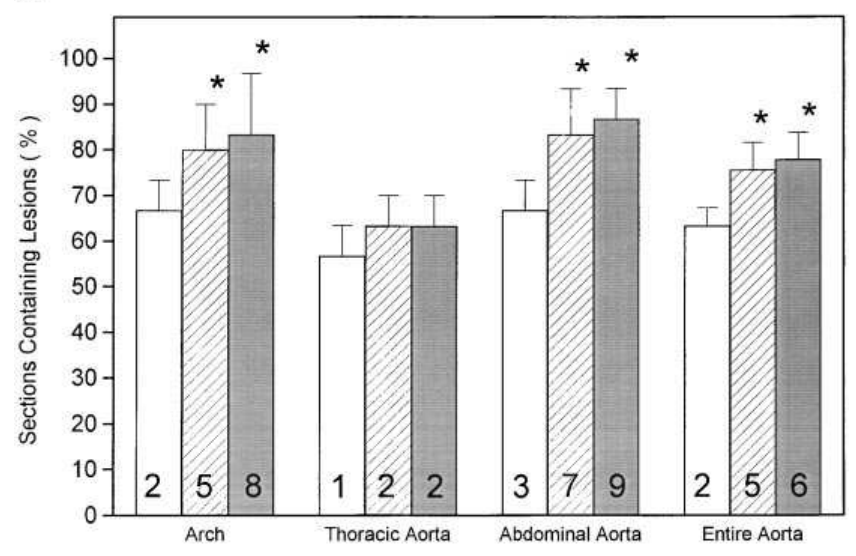

B

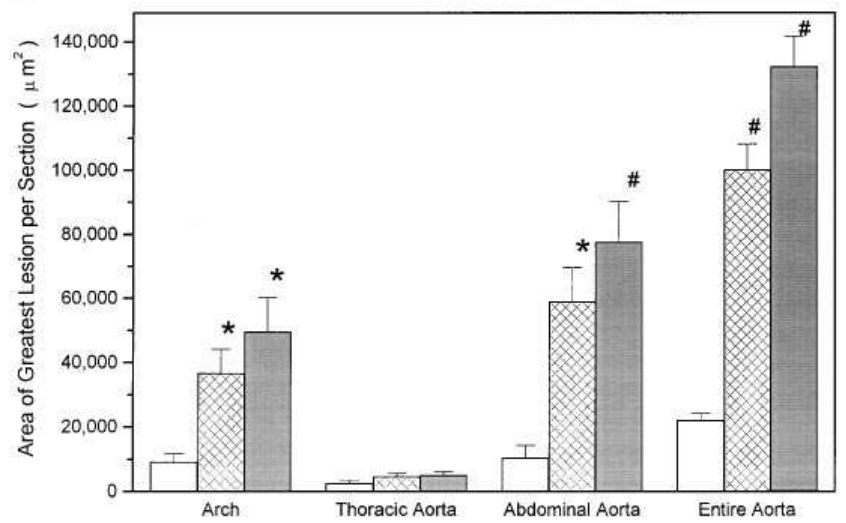

C

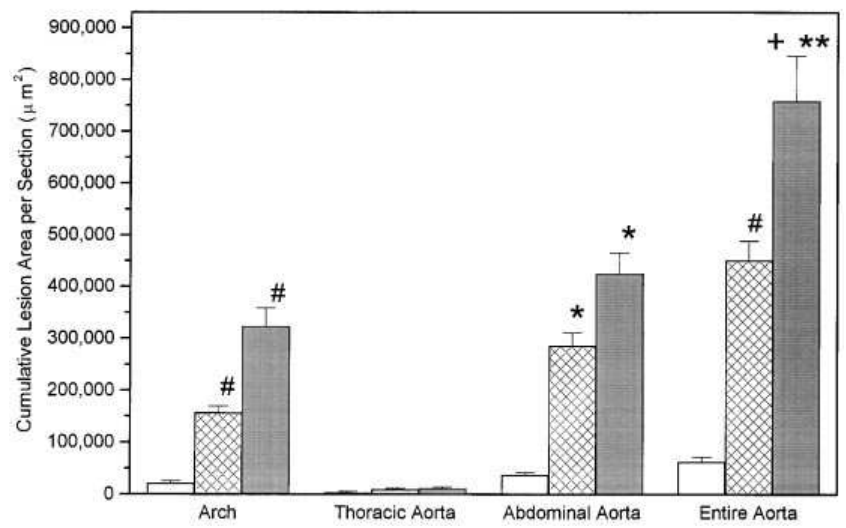

nal segments) were prepared and analyzed by computer-assisted image analysis as described in Methods. (A) Percentage of all sections containing lesions. The average number of lesions (areas of oil red O-stained lipid accumulations) per section is indicated by the insert in the bars. $* P<0.05$ compared with the normocholesterolemic group. $(B)$ Area of the single greatest lesion present in each cross-section.

This parameter provides a conservative measure of lesion formation because small lesions and adaptive intimal thickenings in which lipid and macrophage accumulation is just beginning are ignored. $* P<$ $0.005 ;{ }^{\#} P<0.001$, compared with the normocholesterolemic group. (C) Cumulative area of all oil red $\mathrm{O}$ staining per section. ${ }^{*} P<0.005$; ${ }^{\sharp} P<0.001$; and ${ }^{* *} P<0.0005$ compared with the normocholesterolemic group, respectively; ${ }^{+} P<0.05$ compared with the group with temporary hypercholesterolemia. White bars, normocholesterolemia; hatched bars, temporary hypercholesterolemia; gray bars, hypercholesterolemia. 

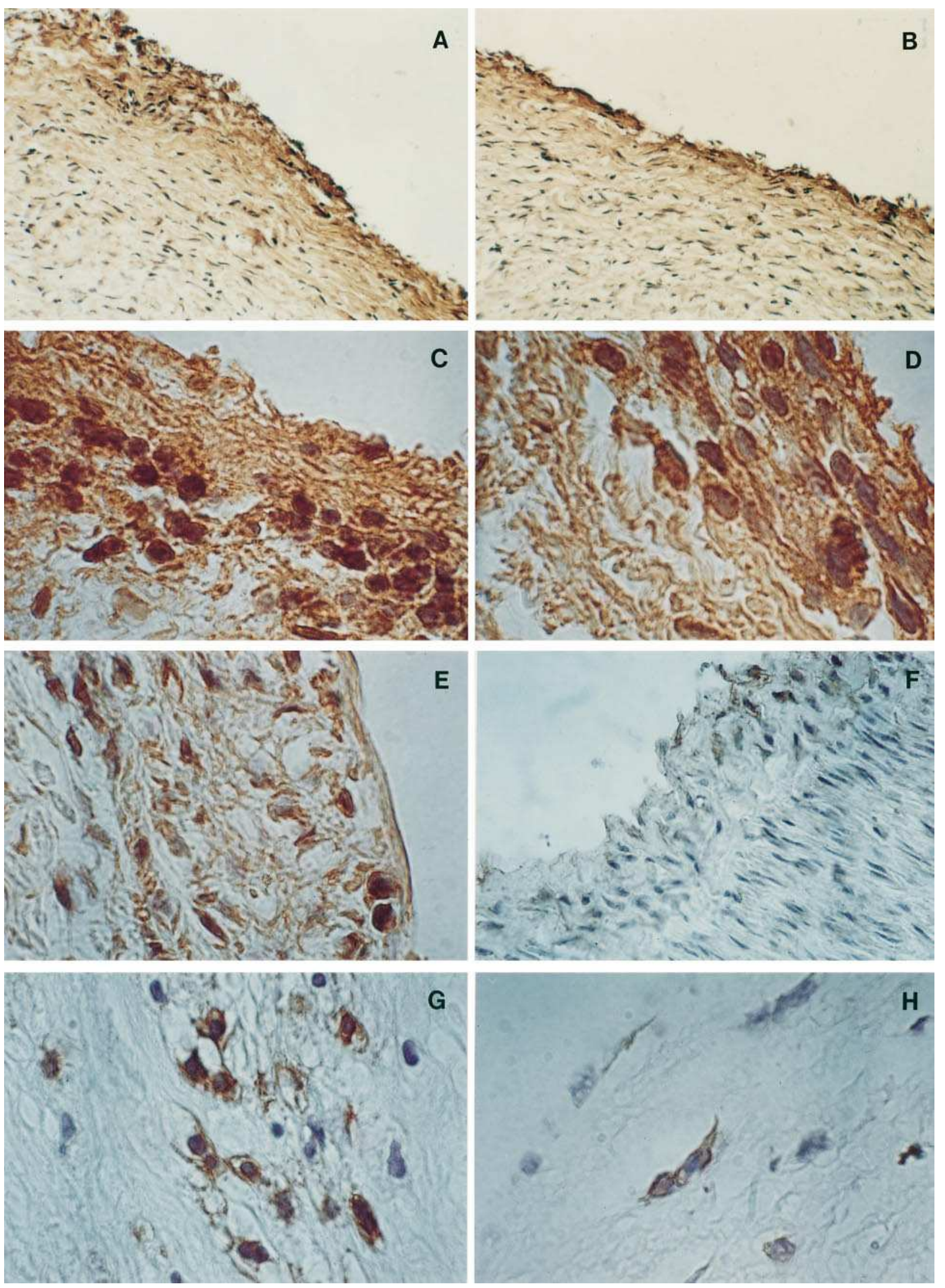

Figure 4. Presence of apo B, oxidation-specific epitopes, and monocyte/macrophages in early lesions of fetal aortas. Tissue sections were prepared as described in Methods and immunostained with the avidin-biotin peroxidase method. Epitopes recognized by the primary antibody are brown; the nuclei are counterstained with hematoxilin. Serial sections of a fetal aorta from the hypercholesterolemic group stained with $(A)$ NP1533975, a monoclonal antibody to human apo B (1:500 dilution), and (B) NA59, a monoclonal antibody against 4-HNE-lysine epitopes 
cept for the cumulative lesion area in the entire aorta $(P<$ $0.05)$, the difference did not reach statistical significance.

To obtain another measure of lesion formation, we also determined intimal/medial ratios in the fetal aortas. Normal adaptive intimal thickening in all three groups had an intimal/ medial ratio of $0.189 \pm 0.95(n=23)$. Minimal lesions containing both OxLDL and macrophage/foam cells had a ratio of $0.325 \pm 0.201(n=65)$. The largest fatty streaks (found mostly in the two hypercholesterolemic groups) had a ratio of $0.755 \pm 0.167(n=28)$.

Occurrence of lipoprotein oxidation in fetal aortas. Serial paraffin-embedded sections of the three aortic segments of the fetal aortas were immunostained with antibodies against macrophages, apo B, and two oxidation-specific epitopes, MDAlysine and 4-HNE-lysine. Both adaptive intimal thickenings undergoing fatty streak formation and established lesions frequently contained apo B in the subendothelial space, as shown in an aortic section from the hypercholesterolemic group stained with NP1533975 (Fig. $4 A$ ). Oxidation-specific epitopes frequently colocalized with apo $\mathrm{B}$, as indicated by Fig. $4 B$, a serial section stained with NA59 (specific for 4-HNE-lysine). The intensity of apo B staining in areas rich in oxidation-specific epitopes, however, varied considerably, as previously observed in humans and animal models of atherosclerosis (data not shown; 22-27). Both oxidation-specific epitopes showed similar distribution (Fig. 4 C, 4-HNE lysine; Fig. $4 D$, MDAlysine), and were mostly found in colocalization with macrophage/foam cells. This result is typical of early stages of lesions in animal models of the disease $(22,23,26,28)$. Lesions from normocholesterolemic mothers were frequently smaller, as indicated in Fig. 3, $B$ and $C$, but generally showed similar staining patterns as lesions of the same size from the two hypercholesterolemic groups (Fig. $4 E$, stained for MDA-lysine). Adaptive intimal thickenings undergoing early atherogenic changes and fatty streaks also contained large numbers of monocyte/macrophages (Fig. 4, $F$ and $G$, stained with HAM56). In addition, isolated monocyte/macrophages were also seen in some adaptive intimal thickenings (Fig. $4 \mathrm{H}$, stained with the antibody against CD11b).

Quantitative analysis of all immunostained sections confirmed these qualitative observations. As shown in Fig. 5, the percentage of aortic sections containing oxidation-specific epitopes, macrophages, and apo B was significantly higher in the hypercholesterolemic groups compared with the normocholesterolemic group, reflecting the greater number and size of lesions previously noted. Within each group, however, the frequency of staining with individual antibodies was similar. Clearly, immunocytochemistry provides only semiquantitative results, and the absolute amounts of different epitopes recognized by different antibodies cannot be compared because

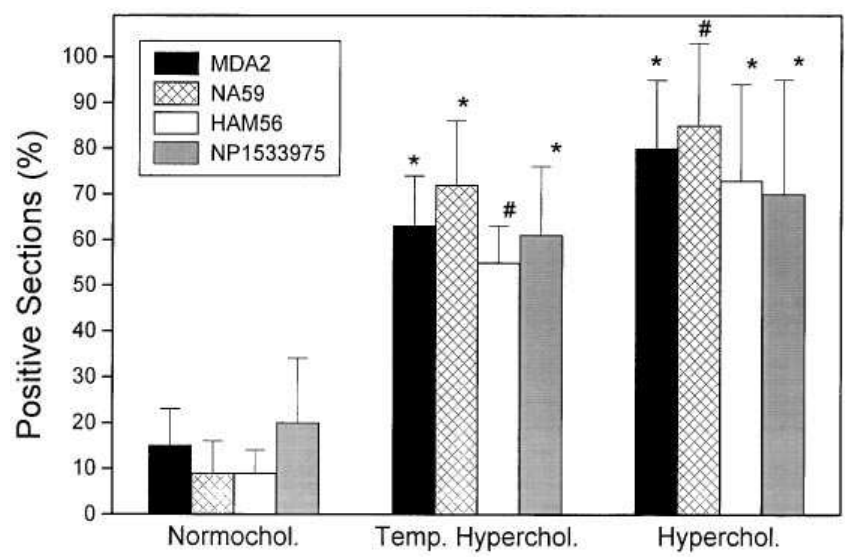

Figure 5. Presence of LDL, oxidation-specific epitopes, and monocyte/macrophages in sections of fetal aortas. Serial sections from the aortic arch and the thoracic and abdominal aorta were immunostained with monoclonal antibodies NP1533975 (against apo B), MDA2 and NA59 (against oxidation-specific epitopes), and HAM56 (against monocyte/macrophages and foam cells) as described in Methods and shown in Fig. 4. Aortic sections containing at least one lesion showing substantial staining were counted as positive, and results were expressed as percent of all sections. ${ }^{*} P<0.005$; ${ }^{\#} P<0.001$ compared with the normocholesterolemic group.

of differences in antibody affinity, epitope accessibility, and other reasons. Nevertheless, the fact that in aortas of the hypercholesterolemic groups, between 63 and $85 \%$ of all sections contained oxidation-specific epitopes, demonstrates that extensive lipid oxidation occurs already during fetal development. Furthermore, the fact that similar percentages of sections contain apo B and oxidation-specific epitopes indicates that LDL is likely to be a primary source of the oxidized lipids.

Differential analysis of aortic lesions. To obtain evidence supporting the hypothesized sequence of events in early atherogenesis, i.e., to verify that LDL infiltration and oxidation precede recruitment of large numbers of monocytes into the vascular intima and their transformation to macrophage/ foam cells, we reexamined the same immunostained serial sections using a different approach. Rather than analyzing areas fitting the traditional definition of early lesions (increased inti$\mathrm{mal} /$ medial ratio, presence of substantial numbers of intimal macrophage/foam cells and lipids), we treated each distinct intimal area that showed positive immunostaining for macrophages, apo B, or oxidation-specific epitopes as a potential atherogenic site. This definition of atherogenic sites was chosen for the purpose of our investigation because it presumably

\section{Figure 4 legend (Continued)}

(1:500 dilution). (C) Higher magnification of a different lesion from the hypercholesterolemic group stained with NA59. Both macrophage-associated and diffuse extracellular presence of oxidation-specific epitopes can be noted. Lesions in aortic sections from the hypercholesterolemic $(D)$ and normocholesterolemic $(E)$ group immunostained with MDA2, a monoclonal antibody against MDA-lysine, another oxidation-specific epitope (1:500 dilution). Staining patterns obtained with MDA2 were generally very similar to those obtained with NA59. $(F)$ Shoulder area of a lesion from the hypercholesterolemic group stained with HAM56, a monoclonal antibody against human monocyte/macrophages (1:500 dilution). Numerous macrophages can be seen in this area that is also rich in lipids (not shown). ( $G$ ) High magnification of an established fatty streak in an aorta from the hypercholesterolemic group stained with HAM56. Numerous macrophages are seen at this site, which also contained OxLDL (not shown). (H) Isolated monocytes stained with anti CD11b (or HAM56, not shown) were found in a small percentage of sites. (Magnification: $A, B, 562.5 \times ; C-E, G, H, 2640 \times ; F, 660 \times$.) 
encompasses the earliest stages of the atherogenic process. It should, however, be kept in mind that not all of these sites will progress to fatty streaks, and that not all fatty streaks will follow the same evolution towards more advanced atherosclerotic lesions. We then examined the same areas in the corresponding serial sections for immunostaining with the other antibodies, and classified the sites into five groups as described in Methods.

Results of the quantitative analysis are reported in Fig. 6. As expected, the vast majority $(58.6 \pm 10.2 \%)$ of all sites contained both oxidation-specific epitopes and apo B (OxLDL in the following), as well as macrophage/foam cells. Most of these sites met the conventional criteria defining fatty streaks. Based on temporal studies in animal models of atherosclerosis in which such lesions appeared after 10-14 d of hypercholesterolemia (40), it can be assumed that these lesions were at least 2 wk old. The other four classes mostly represent sites of lesion initiation, even though some of the sites containing only monocyte/macrophages may also represent normal tissues patrolled by monocytes. A remarkable $17.3 \pm 5.9 \%$ of all atherogenic sites contained only LDL (apo B), and an additional 13.3 $\pm 6.1 \%$ contained only OxLDL (oxidation-specific epitopes and apoB) but no monocyte/macrophages. In contrast, only a very small percentage of sites $(4.3 \pm 2.7 \%)$ contained exclusively macrophages. In most of these sites only isolated cells were seen. In addition, in $6.3 \pm 3.1 \%$ of atherogenic sites both monocyte/ macrophages and native apo B, but few if any oxidation-specific epitopes, were found.

The exact epitope recognized by HAM56 is not known. Al-

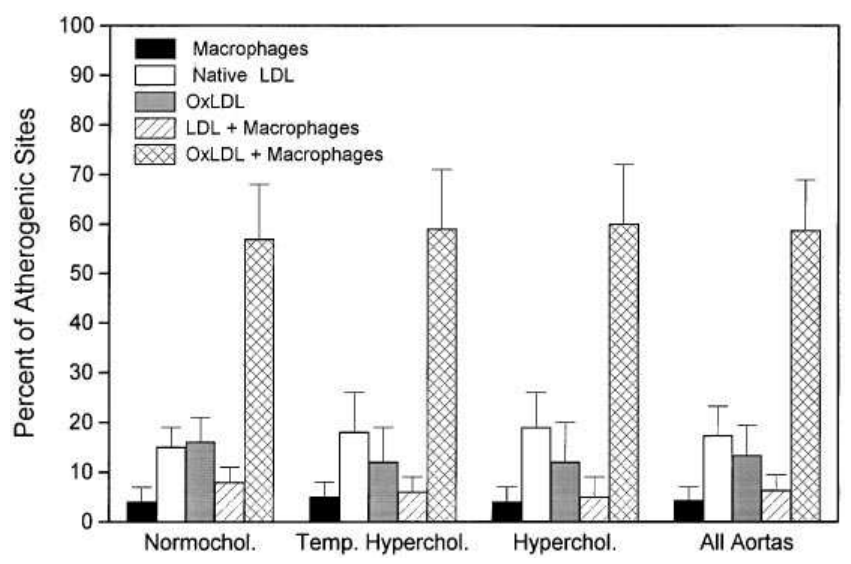

Figure 6. Differential analysis of the composition of atherogenic sites in fetal aortas. Immunostained aortic sections were the same as those used for Fig. 5. For this analysis, an atherogenic site was defined as an intimal area immunostaining with at least one of the antibodies against monocyte/macrophages, apo B, or oxidation-specific epitopes. Each site was then checked in the corresponding serial sections for staining with the other antibodies, and sites were classified into five groups according to results: areas containing only monocyte/ macrophages; areas containing only LDL; areas containing only OxLDL (one or both of the oxidation-specific epitopes and apo B); areas containing LDL and monocyte/macrophages; and areas containing both OxLDL and macrophages or foam cells. Results represent 115 such sites in the normocholesterolemic group, 139 sites in the group with temporary hypercholesterolemia, and 153 in the hypercholesterolemic group. Data were expressed as percent of all sites in the same group. No statistically significant differences were found between groups. though this antibody recognizes monocytes in addition to macrophages, serial sections of some sites were also stained with a second monoclonal antibody against a preserved epitope of monocytes (CD11 $\beta$; Fig. $4 H$ ) to ensure that quantitative analysis was not biased by incomplete detection of monocytes. This procedure yielded very similar results to staining with HAM56 (data not shown).

\section{Discussion}

The primary goal of our systematic analysis of human fetal aortas was to investigate the temporal sequence of events during lesion formation in order to test the hypothesis that intimal accumulation and oxidation of LDL is responsible for recruitment of monocytes and subsequent foam cell formation initiation. In addition, data on the extent of fatty streak formation occurring during early human development would be of value to assess the progression of such lesions to the more advanced lesions seen in young adults, and conversely, to assess their potential for regression. Finally, we hoped to gain insights into the influence of maternal hypercholesterolemia on fetal cholesterol levels and lesion formation.

The first remarkable result was the sheer number of lesions present in fetal aortas. Fetal aortas from normocholesterolemic mothers showed signs of lesion formation in $63.3 \%$ of aortic sections, and even higher percentages were found in the aortas of fetuses from hypercholesterolemic mothers (Fig. $3 A$ ). The prevalence of these lesions in different segments of the aorta reflected the prevalence of fatty streaks and advanced lesions in adults, with the greatest numbers occurring in the abdominal aorta. Maternal hypercholesterolemia dramatically raised the intimal/medial ratios, the cumulative area of intimal lipid accumulations (Fig. $3 \mathrm{C}$ ), and the area of the largest lesion per aortic section (Fig. $3 \mathrm{~B}$ ). For the purpose of this study, we applied the term "lesion" to any thickened intimal area that showed either accumulation of LDL or OxLDL, or macrophage/foam cell accumulation exceeding the presence of isolated monocytes in normal adaptive intimal thickening. Most of these lesions were identifiable only under the microscope, and at best would fit the criteria defining early fatty streaks. It is generally assumed that fatty streaks may progress to more advanced lesions because they occur at the same predilection sites as more advanced lesions (39), and because transitional stages of lesions have been described $(41,42)$. Studies on primates, the experimental model closest to humans, also provide strong evidence for the progression of some fatty streaks to fibrolipid plaques (43). The observation that early lesions are present in a large percentage of cross-sections of fetal aortas suggests that the time of onset of many lesions observed in children and young adults (39) may be much earlier than previously assumed. On the other hand, the sheer extent of lesion formation observed in fetal aortas, and the fact that the size of fetal lesions was strongly related to plasma cholesterol levels that decreased with increasing age, emphasizes the potential for regression of such early lesions. If regression indeed occurs, this could also imply that at early stages of lesion development intimal macrophage/foam cells may regress from the lesion, taking lipid with them. A substantial potential for regression of early lesions would be consistent with the observation that some atherosclerotic lesions of adults can regress, in particular as a result of intensive lipid-lowering intervention (44). 
The fact that large numbers of early lesions were found in fetal aortas may have several explanations. The first of these is the elevated fetal plasma cholesterol level during early fetal development. As indicated by Fig. $1 B$, fetal cholesterol levels showed a significant inverse correlation with fetal age. Presumably, the elevated plasma cholesterol levels observed in younger, more immature fetuses represent a physiological elevation required to meet greater requirements of cholesterol. At the same time, these high cholesterol levels may lead to foam cell formation in the vascular intima. Our observation that fatty streak development was dramatically increased in the two fetal groups with hypercholesterolemic mothers strongly supports the assumption that fetal hypercholesterolemia was an important atherogenic factor.

Although we cannot rule out that the absolute cholesterol levels in the entire group may have been influenced by acute maternal and fetal stress, the striking correlation between fetal plasma cholesterol levels and maternal ones observed in fetuses younger than 6 mo, but not in older ones (Fig. 1C), supports the idea that fetal cholesterol levels before the sixth month of gestation are strongly influenced by maternal cholesterol metabolism. The mechanisms by which this occurs remain to be determined. To date, it has been presumed that fetal cholesterol requirements are met by de novo synthesis rather than by use of maternal or placental cholesterol $(45,38)$. Only limited data, however, are available on fetal lipid parameters during the earlier stages of pregnancy.

A second cause of increased fatty streak formation, particularly in fetuses from hypercholesterolemic mothers, may be a greater sensitivity of fetal aortas to hypercholesterolemia. Our measurement of smooth muscle cell densities in the media provided a clear indication of the immaturity of fetal aortas. Finally, increased lipid peroxidation in the maternal circulation (46) may have enhanced the susceptibility of fetal LDL to oxidation. Other studies have shown that hypercholesterolemia is associated with increased oxidation (30-32); our observation of greater lipid hydroperoxide levels in the plasma of hypercholesterolemic mothers also supports this concept.

One of the goals of this study was to determine whether lipid oxidation occurs in fetal aortas, and if so, whether LDL is a major source of the lipids being oxidized. As described in Results, a very large percentage of all fetal fatty streaks and atherogenic sites contained MDA-lysine and 4-HNE-lysine epitopes. This result is in agreement with our previous observation that lipid fractions extracted from fatty streaks of human fetal aortas contain higher levels of lipid peroxidation products (measured as thiobarbituric acid-reactive substances and conjugated dienes) than lipids extracted from normal areas of the same aortas (47). It should be noted, however, that the monoclonal antibodies used to detect these oxidation-specific epitopes recognize MDA-lysine and 4-HNE-lysine adducts present on a variety of different proteins, and thus are not specific for oxidized apo B $(28,48,49)$. It is possible that some of the immunostaining seen in lesions represents modification of other proteins or cellular membranes by lipid oxidation products. The presence of lipid peroxides in atherosclerotic lesions of human aortas was first detected more than $40 \mathrm{yr}$ ago (50). It has since been established that plaques contain isomers of hydroxy and hydroperoxy cholesterol in esterified and free form (51-53), as well as oxidized fatty acids $(51,53,54)$. Indirect evidence for increased lipid oxidation is also provided by increased titers of circulating autoantibodies to oxidation-spe- cific epitopes in animal models of atherosclerosis $(22,26,28$, $48,49,55)$ and in human patients with increased risk factors or clinical manifestations of atherosclerosis (for review, see reference 56). Lipid oxidation, however, may be involved in a number of pathologies other than atherosclerosis (in particular, diseases associated with inflammatory conditions, such as systemic lupus erythematosus, chronic juvenile arthritis, and eclampsia) where it also gives rise to similar oxidation-dependent neoepitopes (for overview and discussion, see reference 48). To date, it is unknown to what extent oxidation of LDL (or other lipoproteins) contributes to the overall amount of vascular lipid oxidation products. Nevertheless, the fact that in this study we frequently found colocalization between immunostaining with oxidation-specific antibodies and with the monoclonal antibody against apo B suggests that much of the immunostaining obtained with MDA2 and NA59 indeed represents OxLDL. Previous studies combining immunocytochemistry and Western blot analysis of LDL extracted from lesions also support this conclusion (57).

Human studies of LDL oxidation are complicated by the fact that human arteries obtained at autopsy may be subject to spontaneous post-mortem oxidation. Although we used only aortas that were obtained within $3.5 \mathrm{~h}$ after death, and processed the tissues in the presence of strong antioxidant protection, we cannot exclude that the extent of LDL oxidation in vivo may be overestimated to some extent. However, the observation of a very substantial number of lesions containing apo B, but few, if any, MDA- and 4-HNE-lysine epitopes, speaks against extensive postmortem oxidation.

The observation that a large percentage of all atherogenic sites contained only LDL or OxLDL, whereas few lesions contained only macrophages without LDL or OxLDL (Fig. 6), provides strong but indirect evidence for a causal role of LDL accumulation and oxidation in intimal recruitment of monocytes. As expected, the vast majority of atherogenic sites (58.6\%) contained both OxLDL and macrophages. Most of these sites represented established fatty streaks that showed greater intimal/medial ratios than the underlying adaptive intimal thickening. No less than $30.6 \%$ of all atherogenic sites contained only apo B or only OxLDL, but no monocyte/macrophages. In contrast, only $4.3 \%$ of all atherogenic sites contained isolated monocytes in the absence of immunochemically detectable apo B and oxidation-specific epitopes. Even this small percentage is likely to be an overestimation since isolated monocytes observed in some adaptive intimal thickenings may represent tissue patrolling rather than initiation of an atherogenic process.

The fact that $6.3 \%$ of atherogenic sites contained both LDL and monocyte/macrophages, but apparently not OxLDL, may have several explanations. First of all, some of these may represent sites in which intimal LDL accumulation occurs in an area that is being patrolled independently by a few monocytes. The second possibility is that the LDL present at these sites has undergone minimal degrees of oxidation insufficient to generate immunochemically detectable amounts of MDAand 4-HNE-lysine epitopes. Indeed, substantial in vitro evidence suggests that minimally oxidized LDL induces endothelial expression of adhesion molecules and monocyte recruitment (3). Nevertheless, our data cannot rule out that factors independent of LDL and its oxidative modification are responsible for some monocyte recruitment.

Although our results clearly suggest that LDL accumula- 
tion and oxidation precede monocyte recruitment in time, our conclusions about the temporal sequence of events in the initiation of atherogenesis are subject to the limitations of any static observation of a dynamic process. Therefore, we cannot rule out that lesions may also follow several different pathogenetic routes rather than the temporal sequence of events suggested above.

In conclusion, this study demonstrates that early atherogenesis is prevalent in human fetal aortas, and that it is greatly enhanced by maternal hypercholesterolemia. Furthermore, data on lesion composition suggest that intimal LDL accumulation and oxidation are responsible, at least in part, for intimal recruitment of monocytes. This constitutes further support for the role of LDL oxidation in the initiation of atherosclerosis.

\section{Acknowledgments}

Dr. Napoli would like to thank Dr. Giuseppe Ambrosio for his continued encouragement and scientific advice over many years. Dr. D'Armiento would like to thank all Departments of Pediatrics and Gynecology of the Regione Campania that contributed patients or data for their collaboration. The authors also thank Dr. Daniel Steinberg for valuable discussions.

These studies were supported by grant 94.0055 from the Consiglio Nazionale delle Ricerche and Ministero della Università e Ricerca Scientifica e Tecnologica 95/40\% (Faculty of Medicine, Federico II University of Naples), grant ISS.1568 from the Italian National Institutes of Health (C. Napoli), as well as National Heart Lung and Blood Institute grants HL14197 and HL65989 (La Jolla Specialized Center of Research in Molecular Medicine and Arteriosclerosis).

\section{References}

1. Witztum, J.L., and D. Steinberg. 1991. Role of oxidized low density lipoprotein in atherogenesis. J. Clin. Invest. 88:1785-1792.

2. Palinski, W., and J.L. Witztum. 1995. Oxidative stress and diabetes mellitus. In New Horizons In Diabetes Mellitus And Coronary Heart Disease. G.V.R. Born and C.D. Schwartz, editors. Current Science Group, London. 111123.

3. Berliner, J.A., M. Navab, A.M. Fogelman, J.S. Frank, L.L. Demer, P.A. Edwards, A.D. Watson, and A.J. Lusis. 1995. Atherosclerosis: basic mechanisms. Oxidation, inflammation, and genetics. Circulation. 91:2488-2496.

4. Khan, B.V., S. Parthasarathy, R.W. Alexander, and R.M. Medford. 1995. Modified low density lipoprotein and its constituents augment cytokine-activated vascular cell adhesion molecule-1 gene expression in human vascular endothelial cells. J. Clin. Invest. 95:1262-1270.

5. Faruqi, R., C. de la Motte, and P.E. DiCorleto. 1994. Alpha-tocopherol inhibits agonist-induced monocytic cell adhesion to cultured human endothelial cells. J. Clin. Invest. 94:592-600.

6. Fruebis, J., V. Gonzales, M. Silvestre, and W. Palinski. 1997. Effect of probucol treatment on gene expression of VCAM-1, MCP-1 and M-CSF in the aortic wall of LDL receptor-deficient rabbits during early atherogenesis. Arterioscler. Thromb. Vasc. Biol. 17:1289-1302.

7. Radomski, M.W., and S. Moncada. 1993. Regulation of vascular homeostasis by nitric oxide. Thromb. Haemostasis. 70:36-41.

8. Ambrosio, G., C. Napoli, A. Oriente, G. Palumbo, P. Chiariello, G. Marone, M. Condorelli, M. Chiariello, and M. Triggiani. 1994. Oxygen radicals inhibit human plasma acetylhydrolase, the enzyme that catabolizes platelet activating factor. J. Clin. Invest. 93:2408-2416.

9. Carew, T.E., D.C. Schwenke, and D. Steinberg. 1987. Antiatherogenic effect of probucol unrelated to its hypocholesterolemic effect: Evidence that antioxidants in vivo can selectively inhibit low density lipoprotein degradation in macrophage-rich fatty streaks slowing the progression of atherosclerosis in the WHHL rabbit. Proc. Natl. Acad. Sci. USA. 84:7725-7729.

10. Björkhem, I., A. Henriksson-Freyschuss, O. Breuer, U. Diczfalusy, L. Berglund, and P. Henriksson. 1991. The antioxidant butylated hydroxytoluene protects against atherosclerosis. Arterioscler. Thromb. 11:15-22.

11. Sparrow, C.P., T.W. Doebber, J. Olszewski, M.S. Wu, J. Ventre, K.A. Stevens, and Y.S. Chao. 1992. Low density lipoprotein is protected from oxidation and the progression of atherosclerosis is slowed in cholesterol-fed rabbits by the antioxidant N, $\mathrm{N}^{\prime \prime}$-diphenyl-phenylenediamine. J. Clin. Invest. 89:18851891.
12. Sasahara, M., E.W. Raines, A. Chait, T.E. Carew, D. Steinberg, P.W. Wahl, and R. Ross. 1994. Inhibition of hypercholesterolemia-induced atherosclerosis in Macaca Nemestrina by probucol: I. Is the extent of atherosclerosis related to resistance of LDL to oxidation? J. Clin. Invest. 94:155-164.

13. Tangirala, R.K., F. Casanada, J.L. Witztum, D. Steinberg, and W. Palinski. 1995. Effect of the antioxidant $\mathrm{N}, \mathrm{N}^{\prime}$-diphenyl 1,4-phenylenediamine (DPPD) on atherogenesis in apoE-deficient mice. Arterioscler. Thromb. Vasc. Biol. 15:1625-1630.

14. Prasad, K., and J. Kalra. 1993. Oxygen free radicals and hypercholesterolemic atherosclerosis: effect of vitamin E. Am. Heart J. 125:958-973.

15. Fruebis, J., T.E. Carew, and W. Palinski. 1995. Effect of vitamin E on atherogenesis in LDL receptor-deficient rabbits. Atherosclerosis. 117:217-224.

16. Fruebis, J., D. Steinberg, H.A. Dresel, and T.E. Carew. 1994. A comparison of the antiatherogenic effects of probucol and of a structural analog of probucol in LDL-receptor-deficient rabbits. J. Clin. Invest. 94:392-398.

17. Jha, P., M. Flather, E. Lonn, M. Farkouh, and S. Yusuf. 1995. The antioxidant vitamins and cardiovascular disease. A critical review of epidemiologic and clinical trial data. Ann. Intern. Med. 123:860-872.

18. Walldius, G., U. Erikson, A.G. Olsson, L. Bergstrand, K. Hadell, J. Johansson, L. Kaijser, C. Lassvik, J. Molgaard, S. Nilsson, et al. 1994. The effect of probucol on femoral atherosclerosis: the Probucol Quantitative Regression Swedish Trial (PQRST). Am. J. Cardiol. 74:875-883.

19. Kritchevsky, S.B., T. Shimakawa, G.S. Tell, B. Dennis, M. Carpenter, J.H. Eckfeldt, H. Peacher-Ryan, and G. Heiss. 1995. Dietary antioxidants and carotid artery wall thickness. The ARIC Study. Atherosclerosis Risk in Communities Study. Circulation. 92:2142-2150.

20. Azen, S.P., D. Qian, W.J. Mack, A. Sevanian, R.H. Selzer, C.R. Liu, C.H. Liu, and H.N. Hodis. 1996. Effect of supplementary antioxidant vitamin intake on carotid arterial wall intima-media thickness in a controlled clinical trial of cholesterol lowering. Circulation. 94:2369-2372.

21. Heitzer, T., H. Just, and T. Munzel. 1996. Antioxidant vitamin C improves endothelial dysfunction in chronic smokers. Circulation. 94:6-9.

22. Palinski, W., M.E. Rosenfeld, S. Ylä-Herttuala, G.C. Gurtner, S.A Socher, S. Butler, S. Parthasarathy, T.E. Carew, D. Steinberg, and J.L. Witztum. 1989. Low density lipoprotein undergoes oxidative modification in vivo. Proc. Natl. Acad. Sci. USA. 86:1372-1376.

23. Rosenfeld, M.E., W. Palinski, S. Ylä-Herttuala, S. Butler, and J.L. Witztum. 1990. Distribution of oxidation-specific lipid-protein adducts and apolipoprotein B in atherosclerotic lesions of varying severity from WHHL rabbits. Ar teriosclerosis. 10:336-349.

24. Haberland, M.E., D. Fong, and L. Cheng. 1988. Malondialdehyde-altered protein occurs in atheroma of Watanabe heritable hyperlipidemic rabbits. Science. 241:215-241.

25. Boyd, H.C., A.M. Gown, G. Wolfbauer, and A. Chait. 1989. Direct evidence for a protein recognized by a monoclonal antibody against oxidatively modified LDL in atherosclerotic lesions from a Watanabe Heritable Hyperlipidemic rabbit. Am. J. Pathol. 135:815-826.

26. Palinski, W., V. Ord, A.S. Plump, J.L. Breslow, D. Steinberg, and J.L. Witztum. 1994. ApoE-deficient mice are a model of lipoprotein oxidation in atherogenesis: demonstration of oxidation-specific epitopes in lesions and high titers of autoantibodies to malondialdehyde-lysine in serum. Arterioscler. Thromb. 14:605-616.

27. Hammer, A., G. Kager, G. Dohr, H. Rabl, I. Ghassempur, and G. Jürgens. 1995. Generation, characterization, and histochemical application of monoclonal antibodies selectively recognizing oxidatively modified apoB-containing serum lipoproteins. Arterioscler. Thromb. Vasc. Biol. 15:704-713.

28. Palinski, W., S. Hörkkö, E. Miller, U.P. Steinbrecher, H.C. Powell, L.K. Curtiss, and J.L. Witztum. 1996. Cloning of monoclonal autoantibodies to epitopes of oxidized lipoproteins from apoE-deficent mice. Demonstration of epitopes of oxidized LDL in human plasma. J. Clin. Invest. 98:800-814.

29. Stary, H.C. 1992. Composition and classification of human atherosclerotic lesions. Virchows Arch. A Pathol. Anat. Histopathol. 421:277-290.

30. Ohara, Y., T.E. Peterson, and D.G. Harrison. 1993. Hypercholesterolemia increases endothelial superoxide anion production. J. Clin. Invest. 91: 2546-2551.

31. Napoli, C., A. Postiglione, M. Triggiani, G. Corso, G. Palumbo, V. Carbone, A. Ruocco, G. Ambrosio, S. Montefusco, A. Malorni, et al. 1995. Oxidative structural modifications of low density lipoproteins in homozygous familial hypercholesterolemia. Atherosclerosis. 118:259-273.

32. Napoli, C., G. Ambrosio, N. Scarpato, G. Corso, G. Palumbo, F.P. D'Armiento, F.P. Mancini, A. Malorni, S. Formisano, A. Ruocco, and M. Chiariello. 1997. Decreased low-density lipoprotein oxidation after repeated selective apheresis in homozygous familial hypercholesterolemia. Am. Heart J. 133: $585-595$.

33. Mancini, F.P., D.L. Newland, V. Mooser, J. Murata, S. Marcovina, S.G. Young, R.E. Hammer, D.A. Sanan, and H.H. Hobbs. 1995. Relative contributions of apolipoprotein (a) and apolipoprotein B to the development of fatty lesions in the proximal aorta of mice. Arterioscler. Thromb. Vasc.. Biol. 15:19111916.

34. Lillie, R.D. 1965. Histopathologic techniques and practical histochemistry. McGraw Hill, New York.

35. Palinski, W., S. Ylä-Herttuala, M.E. Rosenfeld, S. Butler, S.A. Socher, 
S. Parthasarathy, L.K. Curtiss, and J.L. Witztum. 1990. Antisera and monoclonal antibodies specific for epitopes generated during the oxidative modification of low density lipoprotein. Arteriosclerosis. 10:325-335.

36. Gown, A.M., T. Tsukada, and R. Ross. 1986. Human atherosclerosis: Immunocytochemical analysis of the cellular composition of human atherosclerotic lesions. Am. J. Pathol. 125:191-207.

37. Pei-Zhen, Z., D. Zhuo-Lin, Z. Zhen-Sheng, Z. Hong-Ye, and W. HongYue. 1994. The influence of age and location of arterial lesion on the pathogenesis and development of early atherosclerotic lesions in youth. Chinese Med. J. (Peking). 107:171-175.

38. Neary, R.H., M.D. Kilby, P. Kumpatula, F.L. Game, D. Bhatnagar, P.N. Durrington, and P.M.S. O'Brien. 1995. Fetal and maternal lipoprotein metabolism in human pregnancy. Clin. Sci. (Lond.). 88:311-318.

39. Pathobiological Determinants of Atherosclerosis in Youths (PDAY) Research Group. 1993. Natural history of aortic and coronary atherosclerotic lesions in youth. Findings from the PDAY study. Arterioscler. Thromb. 13: 1291-1298.

40. Schwenke, D.C., and T.E. Carew. 1989. Initiation of atherosclerotic lesions in cholesterol-fed rabbits. I. Focal increases in arterial LDL concentration precede development of fatty streak lesions. Arteriosclerosis. 9:895-907.

41. Stary, H.C., D.H. Blankenhorn, A.B. Chandler, S. Glagov, W. Insull, Jr., M. Richardson, M.E. Rosenfeld, S.A. Schaffer, C.J. Schwartz, W.D. Wagner, and R.W. Wissler. 1992. A definition of the intima of human arteries and of its atherosclerosis-prone regions. A report from the Committee on Vascular Lesions of the Council on Arteriosclerosis, American Heart Association. Circulation. 85:391-405.

42. Stary, H.C., A.B. Chandler, S. Glagov, J.R. Guyton, W. Insull Jr., M.E. Rosenfeld, S.A. Schaffer, C.J. Schwartz, W.D. Wagner, and R.W. Wissler. 1994. A definition of initial, fatty streak, and intermediate lesions of atherosclerosis. A report from the Committee on Vascular Lesions of the Council on Arteriosclerosis, American Heart Association. Circulation. 89:2462-2478.

43. Faggiotto, A., and R. Ross. 1984. Studies of hypercholesterolemia in the nonhuman primate. II Fatty streak conversion to fibrous plaque. Arteriosclerosis. 4:341-356.

44. Blankenhorn, D.H., and H.N. Hodis. 1994. George Lyman Duff Memorial Lecture. Arterial imaging and atherosclerosis reversal. Arterioscler. Thromb. 14:177-192.

45. Belknap, W.M., and J.M. Dietschy. 1988. Sterol synthesis and low density lipoprotein clearance in vivo in the pregnant rat, placenta and fetus. Sources for tissue cholesterol during fetal development. J. Clin. Invest. 82:20772085.

46. Hubel, C.A., J.M. Roberts, R.N. Taylor, T.J. Musci, G.M. Rogers, and M.K. McLaughlin. 1989. Lipid peroxidation in pregnancy: new perspectives on preeclampsia. Am. J. Obstet. Gynecol. 161:1025-1034.
47. D'Armiento, F.P., F. Di Gregorio, S.A. Ciafrè, T. Posca, A. Liguori, C. Napoli, P. Colasanti, A. Calí, and R. Vecchione. 1993. Histological findings and evidence of lipid conjugated dienes and malondialdehyde in human fetal aortas. Acta Paediatr. 82:823-828.

48. Hörkkö, S., E. Miller, E. Dudl, P.D. Reaven, N.J. Zvaifler, R. Terkeltaub, S.S. Pierangeli, L.K. Curtiss, W. Branch, W. Palinski, and J.L. Witztum. 1996. Antiphospholipid antibodies are directed against epitopes of oxidized phospholipids: recognition of cardiolipin by monoclonal antibodies to epitopes of oxidized low-density lipoprotein. J. Clin. Invest. 98:815-825.

49. Hörkkö, S., E. Miller, D.W. Branch, W. Palinski, and J.L. Witztum. 1997. The epitopes for some antiphospholipid antibodies are adducts of oxidized phospholipid and $\beta_{2}$ glycoprotein 1 (and other proteins). Proc. Natl. Acad. Sci. USA. 94:10356-10361.

50. Glavind, J., S. Hartmann, J. Clemmensen, K.E. Jessen, and H. Dam. 1952. Studies on the role of lipoperoxides in human pathology. The presence of peroxidized lipids in the atherosclerotic aorta. Acta Pathol. Microbiol. Scand. 30:1-6.

51. Brooks, C.J.W., G. Steel, J.D. Gilbert, and V.A. Harland. 1971. Lipids in human atheroma. Characterisation of a new group of polar sterol esters from human atherosclerotic plaques. Atherosclerosis. 13:223-237.

52. Chisolm, G.M., G. Ma, K.C. Irwin, L.L. Martin, K.G. Gunderson, L.F. Limberg, D.W. Morel, and P.E. DiCorleto. 1994. 7 $\beta$-Hydroperoxycholest-5-en $3 \beta$-ol, a component of human atherosclerotic lesions, is the primary cytotoxin of oxidized human low density lipoprotein. Proc. Natl. Acad. Sci. USA. 91: 11452-11456.

53. Carpenter, K.L.H., S.E. Taylor, C. Van der Van, B.K. Wilkinson, J.A. Ballantine, and M.J. Mitchinson. 1995. Lipid and oxidised lipids in human atherosclerotic lesions of different stages of development. Biochim. Biophys. Acta. 1256:141-150.

54. Kuhn, H., J. Belkner, R. Wiesner, T. Schewe, V.Z. Lankin, and A.K. Tikhaze. 1992. Structure elucidation of oxygenated lipids in human atherosclerotic lesions. Eicosanoids. 5:17-22.

55. Palinski, W., R.K. Tangirala, E. Miller, S.G. Young, and J.L. Witztum. 1995. Increased autoantibody titers against epitopes of oxidized low density lipoprotein in LDL receptor-deficient mice with increased atherosclerosis. Arterioscler. Thromb. Vasc. Biol. 15:1569-1576.

56. Witztum, J.L., and W. Palinski. 1996. Autoimmunity to oxidized lipoproteins. In Immune Functions of the Vessel Wall. G.K. Hansson and P. Libby, editors. Harwood Academic Publishers, Amsterdam. 159-172.

57. Ylä-Herttuala, S., W. Palinski, M.E. Rosenfeld, S. Parthasarathy, T.E. Carew, S. Butler, J.L. Witztum, and D. Steinberg. 1989. Evidence for the presence of oxidatively modified low density lipoprotein in atherosclerotic lesions of rabbit and man. J. Clin. Invest. 84:1086-1095. 